\title{
Multifractal analysis of the rainfall time distribution on the metropolitan area of Barcelona (Spain)
}

\author{
R. Rodríguez $\cdot$ M. C. Casas $\cdot$ A. Redaño
}

Received: 16 July 2012/ Accepted: 13 April 2013

(C) Springer-Verlag Wien 2013

\begin{abstract}
In most of the studies on scale properties in the rainfall process, multifractal behavior has been investigated without taking into account the different rain generation mechanisms involved. However, it is known that rain processes are related to certain scales, determined by climatological characteristics as well as regional and local meteorological features. One of the implications derived from these correspondences is the possibility that the multifractal parameters of the rainfall could depend on the dominant precipitation generation mechanism. Fractal analysis techniques have been applied in this work to rainfall data recorded in the metropolitan area of Barcelona in the period 1994-2001, as well as to a selection of synoptic rainfall events registered in the same city in the period 1927-1992. The multifractal parameters obtained have been significantly different in each case probably showing the influence of the rain generation mechanisms involved. This influence has been revealed also in the analysis of the effects of seasonality on the multifractal behavior of rainfall in Barcelona.
\end{abstract}

Responsible editor: B. Ahrens.

R. Rodríguez · M. C. Casas $(\bowtie)$

Departament de Física i Enginyeria Nuclear (FEN), EPSEVG, Universitat Politècnica de Catalunya-BarcelonaTech (UPC), Campus Vilanova i la Geltrú-Edif. VG2, Avda. Víctor Balaguer, s/n, 08800 Vilanova i la Geltrú, Spain

e-mail: m.carmen.casas@upc.edu

\section{A. Redaño}

Departament d'Astronomia i Meteorologia (DAM), Facultat de Física, Universitat de Barcelona (UB), Martí i Franqués, 1, 08028 Barcelona, Spain

\section{Introduction}

In mid latitude Mediterranean areas, precipitation is either caused by synoptic scale cloud systems producing rain with low or moderate intensity, or by convective systems much less extensive, belonging to local scale or mesoscale, causing high or moderate intense storms with shorter duration. Even though both kinds of precipitation can be associated with frontal depressions, intense convective systems affecting Mediterranean areas have a particular structure very much influenced by local and seasonal factors, one of the most important being the sea-atmosphere interaction (Codina et al. 1997; Jansà et al. 2001).

The multiplicative cascade model considers large-scale structures smashing to a random number of substructures, with $\lambda$ being the scale relation involved. Rain processes often show multiscaling or multifractal behavior, being a function the governing exponent related to the fractal dimension, instead of the constant exponent corresponding to a simple scale process (Schertzer and Lovejoy 1987; Lovejoy and Schertzer 1990). In most studies on scale properties of the precipitation process, multifractal behavior has been investigated without distinction between observations related to different mechanisms of rainfall generation. However, it is known that rain processes are related to certain scales (Fraedrich and Larnder 1993; Olsson et al. 1993). It can be assumed that there is a scale independence within limits related to properties of rainfall ranging from climatological characteristics to regional and local meteorological properties. An implication that derives from these limitations is that the rainfall multifractal properties can be different for different geographic regions depending on the dominant precipitation generating mechanism (Olsson and Niemczynowicz 1996; de Lima and de Lima 2009). 
In this work, a comparison between multifractal analysis applied on rain data recorded by the urban pluviometric network installed by the company Clavegueram de Barcelona, S.A. (CLABSA) in the urban area of Barcelona (1994-2001) (Enjamio et al. 2005) and a selection of synoptic rainfall events has been made to show up the possible influence of the meteorological scale rain processes on the multifractal parameters obtained. The synoptic events have been selected from the CLABSA records in the same period (1994-2001) and data registered by the Jardí gauge of the Observatori Fabra of Barcelona in the period 1927-1992 (Casas et al. 2004).

\section{Multifractal analysis methodology}

As known, regional and local climatological and meteorological features introduce characteristic scales imposing limits on the theoretically overall scale-independent multifractal approach (Fraedrich and Larnder 1993; Olsson et al. 1993). The scale invariant range or scaling regime has to be evaluated before applying the multifractal analysis. The scaling regime of rainfall time series can be estimated from their power spectrum $E(f)$. If a time series spectrum obeys a power law as (1), where $f$ is the frequency,

$E(f)=f^{-\beta}$

the scaling regime can be identified as approximately a straight-lined zone of the spectrum plotted in a doublelogarithmic diagram. Once this range has been determined, the validity of the multifractal formulation within it has to be investigated.

The scale properties and multifractal behavior of rainfall can be studied through the variation of the statistical moments of the rainfall series with scale (Schertzer and Lovejoy 1987) considering (2),

$\left\langle\varepsilon_{\lambda}^{q}\right\rangle \approx \lambda^{K(q)}$

where $\left\langle\varepsilon_{\lambda}^{q}\right\rangle$ is the ensemble average $q$-order moment of the rainfall series studied at a scale ratio $\lambda$ (ratio between the largest scale of the field and the scale of interest) and $K(q)$ is the moment scaling function. According to the theoretical universal fractal model (Lovejoy and Schertzer 1990), for conservative processes (slope $\beta<1$ in the power spectrum) the function $K(q)$ can be fitted to the following expressions:

$$
\begin{aligned}
& K(q)=\frac{C_{1}}{\alpha-1}\left(q^{\alpha}-q\right), \quad 0 \leq \alpha<1, \quad 1<\alpha \leq 2 \\
& K(q)=C_{1} q \log (q), \quad \alpha=1
\end{aligned}
$$

Using this model, the multifractal behavior can be determined and described only with two parameters: the codimension of the mean process $C_{1}$, related to the rain intermittency or inhomogeneity, and the Lévy index $\alpha$ characterizing the multifractal degree. The double trace moment method (DTM) permits estimating the parameters $\alpha$ and $C_{1}$ using a second $\eta$-order moment (Lavallée et al. 1991)

$K(q, \eta)=\eta^{\alpha} K(q)$

Plotting $K(q, \eta)$ as a function of $\eta$ in a $\log -\log$ diagram, the slope of the straight-line zone gives an estimation of the parameter $\alpha$. Then, constant $C_{1}$ can be obtained from (3).

For moments of order $\mathrm{q}$ higher than a critical $q_{\mathrm{D}}$, the empirical function $K(q)$ shows linear behavior. This discontinuity of the scale function is due to a divergence of moments for $q>q_{\mathrm{D}}$, which can also be detected in the power dependence shown in the empirical probabilistic distribution function tails of the process; the exponent corresponds approximately to the critical moment $q_{\mathrm{D}}$.

\section{Rainfall data and selection of the synoptic rainfall events}

The urban rain gauge network of Barcelona managed by CLABSA, in operation since 1994, is at the moment constituted by 23 rain gauges, tilting-bucket type with a resolution of $0.1 \mathrm{~mm}$, distributed over an area of $100 \mathrm{~km}^{2}$. The continuous records of 22 of the pluviometers are available from 1996 to 2009. Five of these pluviometers started working in 1994 and there is a 23rd gauge operating from 2000. The company provides reports of anomalies and revision periods to be taken into account. From records in the period 1994-2001, a total of 157 annual series of 8-min accumulated rainfall amounts have been obtained to be analyzed using the multifractal technique. Seasonal subsets from these series have been considered to study the effects of seasonality on the multifractal behavior of rainfall in Barcelona.

To determine the possible influence of the kind of rainfall registered on the multifractal analysis results, data corresponding to synoptic rainfall events observed in Barcelona in the period 1927-2001 were selected. In a previous work, Casas et al. (2004) applied a cluster analysis to 44 intense rain events recorded by the Jardí gauge located in the Observatori Fabra of Barcelona showing a return period equal or larger than 5 years for any duration between $5 \mathrm{~min}$ and $24 \mathrm{~h}$. The objective classification of these storms depending on the scale of the meteorological process involved in their origin was obtained. The synoptic group was constituted by nine events with intensities exceeding the 5-year return period only for the 12 and 24-h interval. The same methodology was applied later to the urban network CLABSA series in the period 1994-2001 
(Casas et al. 2010) and three synoptic rainfall episodes were found. In almost every case, a mid troposphere low located in the SW of the Iberian Peninsula was present and no seasonal influence was observed. Considering the network records corresponding to these three synoptic storms, a total of 51 rainfall events were obtained. The mean duration of these events is $19 \mathrm{~h}$ (corresponding approximately to 143 amounts of. 8-min accumulated rainfall). Considering the 9 synoptic episodes registered by the Jardí gauge and the 51 events recorded by the urban network, the synoptic sample to be analyzed using the multifractal procedure was constructed with a total of 60 series of 128 amounts of 8-min accumulated rainfall.

\section{Multifractal analysis of the rainfall data}

To determine the scaling regimes present in the selected data, the average power spectrum of the 157 annual series of 8-min accumulated rainfall amounts from the urban network records was calculated. Considering this power spectrum, a smoothed distribution was obtained averaging over logarithmically spaced frequency intervals. From 16 min to almost $1 \mathrm{~h}$, a power regime with exponent $\beta \approx 1$ can be identified (Fig. 1). Despite the agreement of this temporal range with rainfall generated by micro and mesoscale processes, Fraedrich and Larnder (1993) are skeptical with respect to its meteorological nature arguing possible measurement instrument deficiencies related with the inability of the gauges to measure low rain intensities. Olsson (1995) proved this assumption by showing that on increasing the intensity resolution of the data $(0.004 \mathrm{~mm} /$ $\min$ ), this power regime disappeared. Data recorded by the tipping bucket pluviometers of the urban network of Barcelona have a lower-intensity resolution (approximately $0.01 \mathrm{~mm} / \mathrm{min}$ ) and therefore this low-frequency break appears.

From $1 \mathrm{~h}$ to several weeks, a transition regime was observed (Fig. 1). This transition regime is very similar to that obtained by Fraedrich and Larnder (1993) for a 5-min data series recorded at Postdam station (Germany). In accordance with Fraedrich and Larnder (1993), we interpret this regime as an overlapping region between two scaling potential behaviors: first, a regime with a slope of $\beta \approx 0.5$ in the temporal range which corresponds to frontal systems and other synoptic systems producing rainfall from several hours to days, and second, the flat region with slope 0 or plateau associated with seasonal rain variability corresponding to several months' range. This overlapping complicates the determination of an upper limit of the regime associated with synoptic systems.

As expected, power dependence in the empirical probabilistic distribution tails were observed. The series of

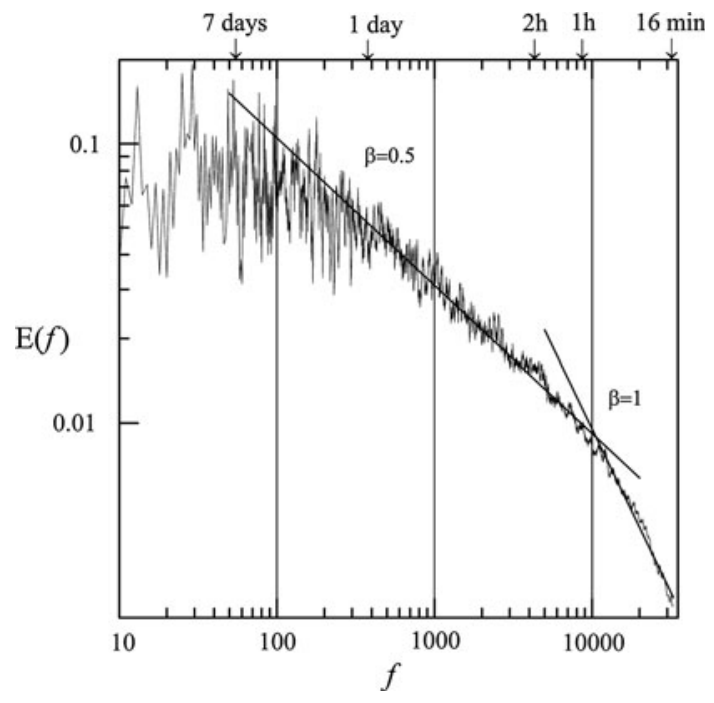

Fig. 1 Power spectrum $E(f)$ of the urban network rainfall series averaged over logarithmically spaced frequency intervals. Two power law behaviors can be identified (straight lines fitted by linear regression)

8-min accumulated rainfall amounts was sorted in ascending order and the empirical probability of exceedance of every value $X_{i}$ calculated using the Weibull formula (5)

$\operatorname{Pr}\left(X<X_{i}\right)=1-\frac{i}{n+1}$

where $i$ is the assigned rank to the rainfall amount $X_{i}$ and $n$ is the total number of observations. The equation proposed by Andrade et al. (1998) (6), widely used in bibliography, was used to fit the tail of the empirical probabilistic distribution function:

$\operatorname{Pr}\left(X<X_{i}\right) \sim \frac{1}{X^{q_{\mathrm{D}}}}\left[1-\left(\frac{X}{M}\right)^{q_{\mathrm{D}}}\right]$

with $M$ being the maximum $X$ observed and $q_{\mathrm{D}}$ the critical moment. Equation (6) was fitted to the 36 points with intensities higher than that corresponding to a return period of 1 year. A value of $q_{\mathrm{D}}=4.1$ was estimated (see Fig. 2). As reported by Douglas and Barros (2003), the most common range values of $q_{\mathrm{D}}$ extend between a little less than 2 and slightly more than 3 . Other studies have found values of $q_{\mathrm{D}}$ between 1.7 and 5 (Fraedrich and Larnder 1993; Labat et al. 2002). This variation has been attributed also to measurement limitations related to time resolution of the observations and length of the series (Olsson 1995).

To characterize the multifractal behavior of rainfall in Barcelona, the statistical moments of the considered set of data, as well as their scale properties, were analyzed. A range between $8 \mathrm{~min}\left(2^{3} \mathrm{~min}, \lambda=2^{16}\right)$ and 1 year (approximately $2^{19} \mathrm{~min}, \lambda=1$ ) was considered for analysis. Moments from $q=0.1$ to $q=5.0$ were calculated for 


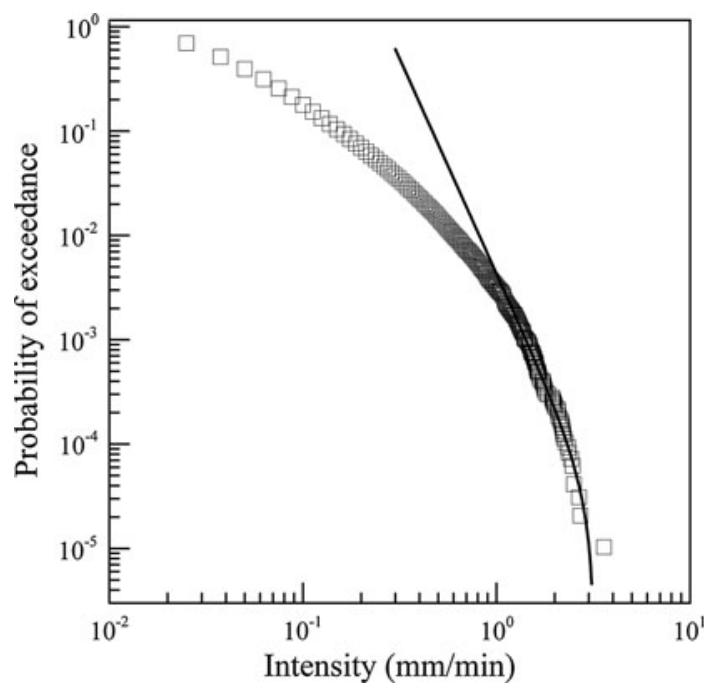

Fig. 2 Empirical probability of exceedance of the rainfall intensity. Tail values were fitted to Eq. (6) with an estimated value of the critical moment $q_{\mathrm{D}}=4.1$

scales from $\lambda=65,536$ ( $8 \mathrm{~min})$ to $\lambda=2$ (182 days). From $8 \min (\lambda=65,536)$ to 22.8 days $(\lambda=16)$, linear regressions were made, resulting in all the correlation coefficients being higher than 0.99 . Some of these linear regressions are shown in Fig. 3. For small time scales $(\lambda \geq 4,096$, i.e., $2 \mathrm{~h}$ ), deviations from the power law of Eq. (2) were observed. This behavior is compatible with the linear regime appearing in the Fourier spectrum for time shorter than $1 \mathrm{~h}$ attributed to the low resolution of the instruments. Figure 4 shows the scaling function values calculated for the network series, for every $q$-order moment. These values outline a slightly convex curve until the critical value $q_{\mathrm{D}}=4.1$, appearing then as a linear dependence compatible with the expected divergence of moments. Making use of the DTM method (Lavallée et al. 1991), curves fitting the theoretical model (3) were obtained (Fig. 5). The curves exhibit a straight-line behavior for a range from $q \cdot \eta=1.2$ to $q \cdot \eta=4$ in which straight lines were fitted by regression. Parameter $\alpha$ was estimated as the average slope of these straight lines for $q$ between 1.5 and 3, which is 0.58 with a standard deviation of 0.04 . From this value of $\alpha=0.58 \pm 0.04$, the parameter $C_{1}$ becomes $C_{1}=0.49 \pm$ 0.02 (Table 1). The discrepancy observed between the data and the theoretical curve in Fig. 4 for $K(q)<0$ is due to the presence of zero values in the data series, not considered in the theoretical model. The parameter values obtained resemble those found by other authors (de Lima and Grasman 1999; Ladoy et al. 1993) in zones with similar meteorological characteristics.

An important dispersion of the multifractal parameters obtained for rainfall processes can be found in bibliography. For instance, there are $\alpha$ estimations in the range from 0.4 to 1.3 for the time series (Hubert et al. 1993, Pathirana

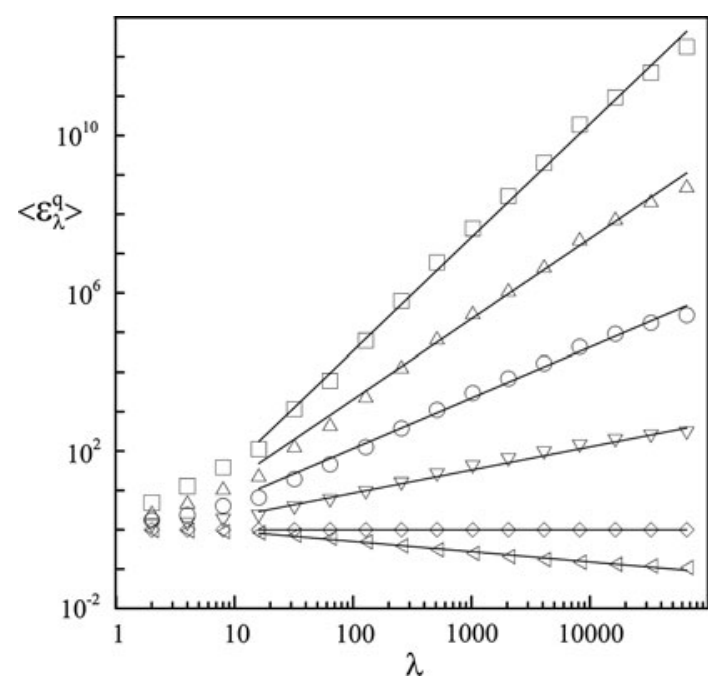

Fig. 3 Log-log plot of the statistical moments q of the urban network rainfall series versus the scale ratio $\lambda$, from $8 \min (\lambda=65,536)$ to 182 days $(\lambda=2)$. From top to bottom $q=5,4,3,2,1$ and 0.5 . Straight lines indicating scale invariance have been fitted by regression from 8 min to 22.8 days

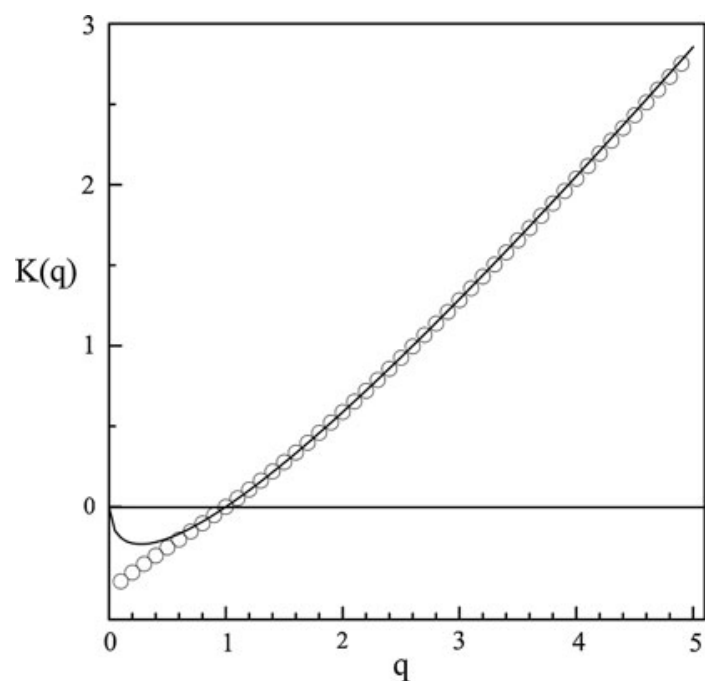

Fig. 4 Empirical scaling function $K(q)$ for the network rainfall series and theoretical curves corresponding to the universal multifractal model obtained using the DTM method (continuous curve). Discrepancy observed for $K(q)<0$ is due to the presence of zero values in the data series, not considered in the theoretical model

et al. 2003) and a range from 0.9 to 1.7 for spatial analysis (Tessier et al. 1993, Olsson and Niemczynowicz 1996). Since the universal multifractal model assumes universal parameters values, this variation has been attributed to the different nature of the data sets involved and the limited sample sizes used (Lovejoy and Schertzer 1995). However, this variability seems to be related to actual climatic discrepancies between different geographic zones. So, restricting the analysis to the European area, the parameter $\alpha$ obtained for Mediterranean and semi-arid locations is 


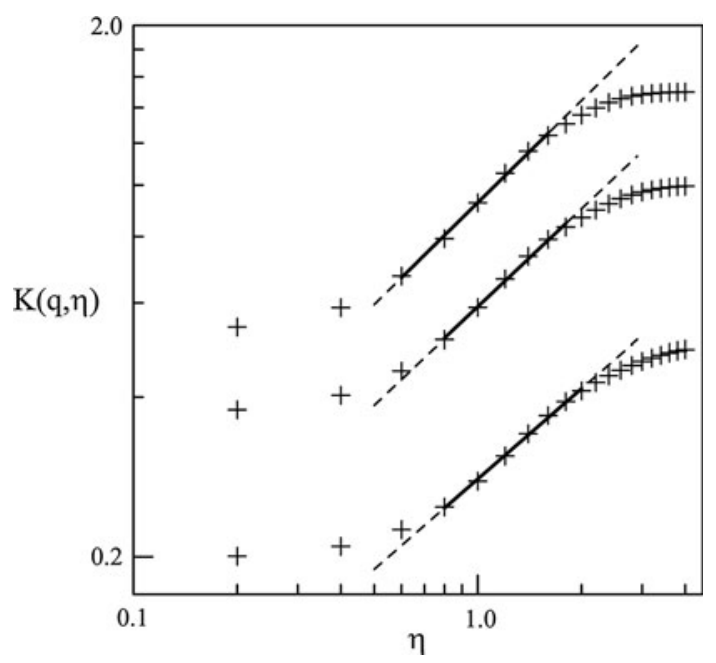

Fig. 5 Log- $\log$ plot of the empirical scaling function $K(q, \eta)$ versus $\eta$ for the network rainfall series and, from top to bottom, for $q=2.5,2$ and 1.5. For $\eta q<q_{\mathrm{D}}=4.1$ the straight lines have a slope which corresponds to $\alpha=0.58 \pm 0.04$. A value of $C_{1}=0.49 \pm 0.02$ was estimated

Table 1 Multifractal parameters $\alpha$ and $C_{1}$ estimated for rainfall in Barcelona

\begin{tabular}{lll}
\hline & $\alpha$ & $C_{1}$ \\
\hline Total set & $0.58 \pm 0.04$ & $0.49 \pm 0.02$ \\
Summer subset & $0.63 \pm 0.05$ & $0.49 \pm 0.01$ \\
Winter subset & $0.96 \pm 0.07$ & $0.29 \pm 0.02$ \\
Synoptic subset & $1.23 \pm 0.03$ & $0.14 \pm 0.01$ \\
\hline
\end{tabular}

usually lower than for rainy and humid areas, whereas $C_{1}$ is greater. For instance, the parameters estimated by Ladoy et al. (1993) for Nîmes (France) were $\alpha=0.45$ and $C_{1}=0.6$, and de Lima and Grasman (1999) found $\alpha=0.49$ and $C_{1}=0.51$ for Vale Formoso (Portugal). In Spain, Valencia et al. (2010) estimated $\alpha=0.55-0.57$ and $C_{1}=0.38-0.39$ as the mean values of these parameters for 14 meteorological stations located in the Ebro River Basin, and García-Marín et al. (2013) obtained $\alpha=0.553$ and $C_{1}=0.358$ for Málaga. On the other hand, the parameters estimated for humid Atlantic climate locations as Madeira (de Lima and de Lima 2009) were $\alpha=0.71$ and $C_{1}=0.34$, and for Valentia (southwestern Ireland) Kiely and Ivanova (1999) estimated $C_{1}$ in the range 0.30-0.34. Labat et al. (2002) found $\alpha=0.9$ and $C_{1}=0.2$ for Ariège (French Pyrenées) and Olsson (1995) obtained $\alpha=0.63$ and $C_{1}=0.44$ for Lund (southern Sweden). In their study using daily records from 30 stations all over the French territory, Tessier et al. (1996) found $\alpha=0.7$ and $C_{1}=0.4$, whereas Biaou (2004) estimated the average values of $\alpha=0.74$ and $C_{1}=0.4$ using 6-min accumulated rainfall series from 243 French stations.
To determine the possible influence of the different rainfall generating mechanisms involved in the multifractal parameters, the same methodology was applied to the synoptic data set mentioned in Sect. 3. From synoptic rainfall events registered in Barcelona by the Jardí gauge and the urban network in the period 1927-2001 [objectively classified as synoptic by Casas et al. (2004) and (2010)], a set of 60 series of 128 amounts of 8-min accumulated rainfall was constructed as a synoptic sample to be analyzed using the multifractal procedure. The analysis of this synoptic data set shows that the scaling range stands up to $17 \mathrm{~h}$ (from $\lambda=1$ to $\lambda=128$ ) (Fig. 6). The best fitting parameters calculated for these synoptic rainfall series making use of the DTM method are $\alpha=1.23 \pm 0.03$ and $C_{1}=0.14 \pm 0.01$ (Figs. 7, 8). Notice that the discrepancy between the empirical scaling moment function $K(q)$ and the DTM theoretical curve for $K(q)<0$ due to the presence of zero values in the data series is not observed for the synoptic rainfall (big squares in Fig. 8), since the selection of the synoptic data has restricted the presence of zero values to some small inside intervals of zero rainfall that disappear in the aggregation process approximately from $1 \mathrm{~h}$ $(\lambda=8,192)$. Parameter $\alpha$ resulted clearly higher (1.23) for that selected synoptic rainfall data set than those calculated for the original series $(0.58)$ with predominant convective rainfall. This result is concordant with that obtained by Olsson and Niemczynowicz (1996) in their spatial analysis over southern Sweden, where $\alpha$ was found also to be lower for convective precipitation than for warm fronts rainfall. Parameter $C_{1}$ points signal intermittency, so a smaller value was obtained for the persistent and smooth precipitation corresponding to the synoptic scale $(0.14)$ than that obtained for total rainfall (0.49). In fact, the use of sequences of continuous rain (without zeros) led some researchers to estimate high values of $\alpha$ and very low values of $C_{1}$. Thus, de Montera (2009), using data collected during the African monsoon in summer 2006 in Benin, and Ghanmi et al. (2012), in the semi-arid region of Grand-Tunis, estimated $\alpha$ in a range from 1.3 to 1.6 , and $C_{1}$ between 0.13 and 0.15 .

A similar result is found when rainfall data are seasonally analyzed. A substantial percentage of the total rainfall amount in Barcelona (and Catalonia in general) stems from convective cloud systems. Llasat and Puigcerver (1997) found that the ratio of convective to total rainfall amounts ranges from 70 to $80 \%$ in the summer months to $<30 \%$ in winter. The effects of seasonality on the multifractal behavior of the rainfall data were analyzed by applying the same methodology to the summer and winter subseries of 8-min accumulated rainfall amounts. The parameters found are listed in Table 1. The winter subseries, with less percentage of convective rainfall, show a multifractal behavior very similar to that obtained for the synoptic subset ( $\alpha=0.96 \pm 0.07$ and $\left.C_{1}=0.29 \pm 0.02\right)$, whereas results for the summer subseries are very similar to those obtained 


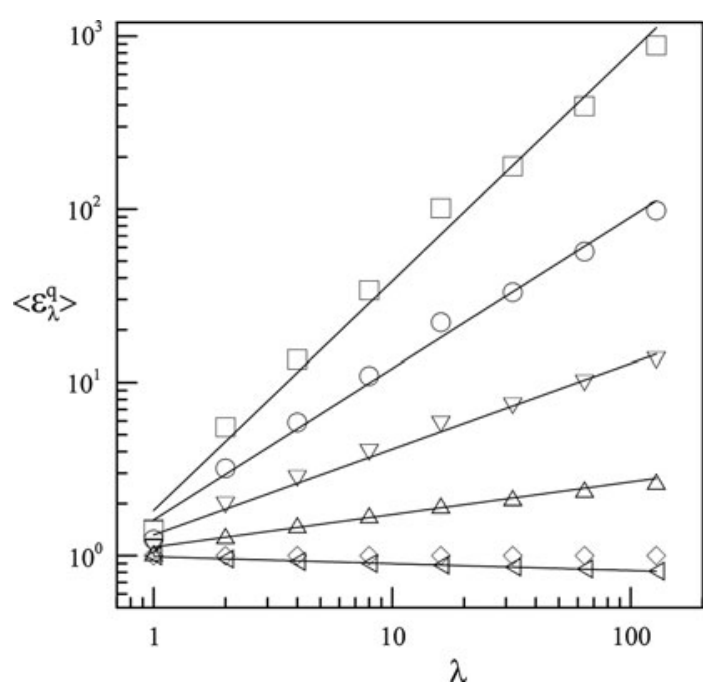

Fig. $6 \mathrm{Log}-\log$ plot of the statistical moments $\mathrm{q}$ of the synoptic rainfall data set versus the scale ratio $\lambda$, from $8 \min (\lambda=128)$ to $17 \mathrm{~h}$ $(\lambda=1)$. From top to bottom $q=5,4,3,2,1$ and 0.5. Straight lines indicating scale invariance were fitted by regression from $8 \mathrm{~min}$ to $17 \mathrm{~h}$

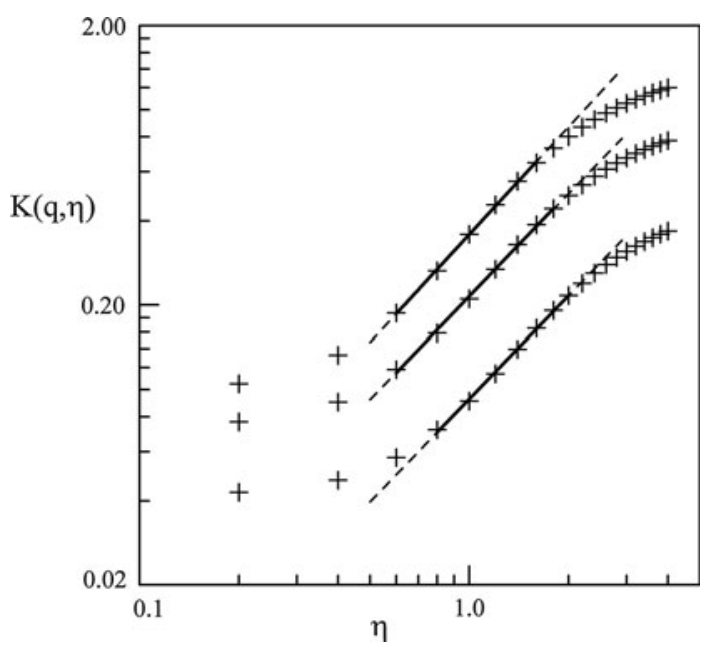

Fig. 7 Log- $\log$ plot of the empirical scaling function $K(q, \eta)$ versus $\eta$ for the synoptic rainfall data set and, from top to bottom, for $q=2.5$, 2 and 1.5. For $1.5<\eta q<4.1$, the straight lines have a slope which corresponds to $\alpha=1.23 \pm 0.04$. A value of $C_{1}=0.14 \pm 0.01$ was estimated

for the total rainfall series $(\alpha=0.63 \pm 0.05$ and $C_{1}=0.49 \pm 0.01$ ). Figure 8 compares the scaling functions corresponding to each of the rainfall samples considered.

\section{Conclusions}

The analysis of the rainfall scaling regime in Barcelona has revealed: a first interval from 16 minutes to $1 \mathrm{~h}$ attributed, despite its temporal agreement with micro and mesoscale

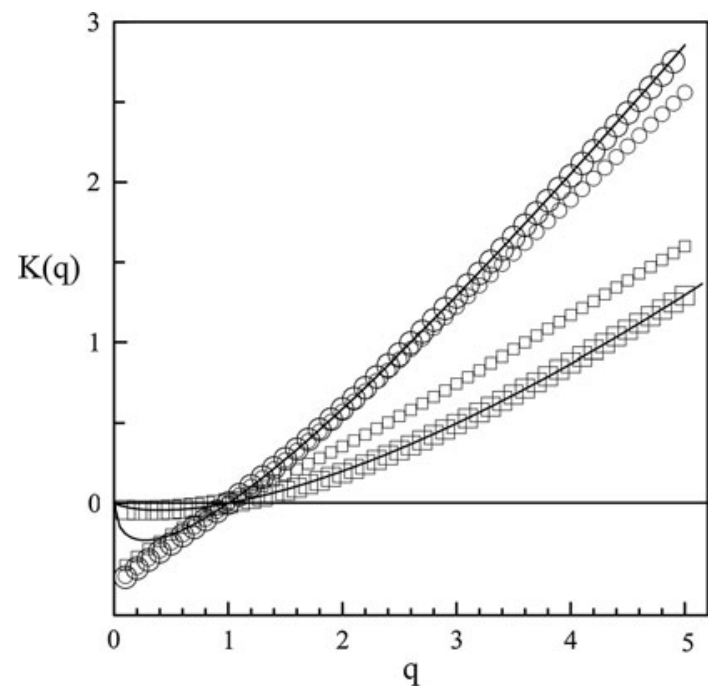

Fig. 8 Empirical scaling function $K(q)$ for the total rainfall data set (big circles) and several subsets: synoptic rainfall (big squares), summer rainfall (small circles) and winter rainfall (small squares). The theoretical curves fitted to the total and synoptic data sets have been represented. There is no discrepancy between $K(q)$ and the synoptic curve for $K(q)<0$ because this subset only presents small inside intervals of zero rainfall that disappear in the aggregation process from approximately $1 \mathrm{~h}$ onward

rainfall processes, to the low resolution of the measurement instruments; a second range from $1 \mathrm{~h}$ to several weeks interpreted as an overlapping zone between the temporal interval corresponding to synoptic rain (from several hours to several days) and the plateau associated with seasonal rain variability. To characterize the multifractal behavior of rainfall, a moment scaling function $K(q)$ was estimated for the scaling range from $8 \mathrm{~min}$ to 22.8 days. The slightly convex appearance of the $K(q)$ function until the critical value $q_{\mathrm{D}}=4.1$ shows a multifractal structure involved. The DTM method has been used to fit the $K(q)$ function to the theoretical universal fractal model formulation. An expected discrepancy between the empirical and the theoretical function for $K(q)<0$, due to the presence of numerous zero values in the analyzed series, was observed. The fitting parameters obtained, $\alpha=0.58 \pm 0.04$ and $C_{1}=0.49 \pm 0.02$, are similar to those calculated by other investigators in similar meteorological zones. The application of the same methodology to a synoptic rainfall data set registered in Barcelona in the studied period has shown up the possible influence of the different rainfall generating mechanisms on the multifractal parameters obtained. The fitting parameters of the scaling function $K(q)$ have been different for total rainfall (mesoscale processes predominantly) and synoptic rainfall, with $\alpha$ being much higher for the synoptic data set (1.23) than for total rainfall (0.58). Parameter $C_{1}$, related to inhomogeneity and signal intermittency, was high for total rainfall (0.49) and significantly 
smaller for the more persistent and smooth rain corresponding to the synoptic scale (0.14). A seasonal analysis was also performed: results seem to corroborate the possible influence of the rainfall generating mechanisms on the multifractal behavior. Parameters $\alpha$ and $C_{1}$ for winter (less of $30 \%$ of convective rainfall $)$ were similar $(\alpha=0.96$, $\left.C_{1}=0.29\right)$ to those obtained for the synoptic subset, whereas for summer (70-80\% of convective rainfall) the parameters were very close $\left(\alpha=0.63, C_{1}=0.49\right)$ to those corresponding to the total rainfall sample.

Acknowledgments This work was supported by the Spanish Ministry of Science and Innovation under Grant CGL2007-65891-C0503. The authors are also grateful to Clavegueram de Barcelona S.A. (CLABSA) for providing the rainfall data used in this study.

\section{References}

Andrade RFS, Schellnhuber HJ, Claussen M (1998) Analysis of rainfall records: possible relation to self-organized criticality. Phys A 254:557-568

Biaou A (2004) Agrégation/désagrégation spatio-temporelle des champs de précipitation. PhD Thesis, U. P. \& M. Curie, Paris

Casas MC, Codina B, Redaño A, Lorente J (2004) A methodology to classify extreme rainfall events in the western Mediterranean area. Theoret Appl Climatol 77:139-150

Casas MC, Rodríguez R, Redaño A (2010) Analysis of extreme rainfall in Barcelona using a microscale rain gauge network. Meteorol Appl 17:117-123. doi:10.1002/met.166

Codina B, Aran M, Young S, Redaño A (1997) Prediction of a mesoscale convective system over Catalonia (Northeastern Spain) with a nested numerical model. Meteorol Atmos Phys 62(1-2):9-22

de Lima MIP, de Lima JLMP (2009) Investigating the multifractality of point precipitation in the Madeira archipelago. Nonlinear Processes Geophys Euro Geosci Un (EGU) Am Geophy Un (AGU) 16:299-311 doi:10.5194/npg-16-299-2009

de Lima MIP, Grasman J (1999) Multifractal analysis of 15-min and daily rainfall from semi-arid region in Portugal. J Hydrol 220:1-11

de Montera L, Barthes L, Mallet C (2009) The effect of rain-no rain intermittency on the estimation of the universal multifractal model parameters. J Hydrometeorology 10:493-506

Douglas E, Barros AP (2003) Probable maximum precipitation estimation using multifractals: application in the eastern United States. J Hydrometeorology 4(6):1012-1024

Enjamio C, Vilar E, Redaño A, Fontán FP, Ndzi D (2005) Experimental analysis of microscale rain cells and their dynamic evolution. Radio Science 40, RS3015. doi: 10.1029/2004 RS003119

Fraedrich K, Larnder C (1993) Scaling regimes of composite rainfall time series. Tellus 45(A):289-298

García-Marín AP, Ayuso-Muñoz JL, Jiménez-Hornero FJ, Estévez J (2013) Selecting the best IDF model by using the multifractal approach. Hydrol Process 27:433-443. doi:10.1002/hyp.9272
Ghanmi H, Mallet C, Verrier S, Barthes L, Bargaoui Z (2012) Study of rainfall intensities for micro-scales in a semi-arid zone (Tunis) by FIF model. In: 3rd STAHY International Workshop on Statistical Methods for Hydrology and Water Resources Management, Tunis, Tunisia

Hubert P, Tessier Y, Lovejoy S, Schertzer D, Schmitt F, Ladoy P, Carbonnel JP, Violette S, Desurosne I (1993) Multifractals and extreme rainfall events. Geophys Res Lett 20:931-934

Jansà A, Genovés A, Picornell MA, Campins J, Riosalido R, Carretero O (2001) Western Mediterranean cyclones and heavy rain. Part 2: statistical approach. Meteorol Appl 8(1):43-56

Kiely G, Ivanova K (1999) Multifractal analysis of hourly precipitation. Phys Chem Earth Part B hydrol Ocean Atmosphere 24(7):781-786. doi:10.1016/S1464-1909(99)00080-5

Labat D, Mangin A, Ababou R (2002) Rainfall-runoff relations for karstic springs: multifractal analysis. J Hydrol 256:176-195

Ladoy P, Schmitt F, Schertzer D, Lovejoy S (1993) Variabilité temporelle multifractale des observations pluviométriques à Nîmes. C R Acad Sci Paris II 317:775-782

Lavallée D, Schertzer D, Lovejoy S (1991) On the determination of the co-dimension function. In: Schertzer D, Lovejoy S (eds) Scaling fractals and non-linear variability in geophysics. Kluwer, Dordrecht, pp 99-110

Llasat MC, Puigcerver M (1997) Total rainfall and convective rainfall in Catalonia. Spain Int J Climatol 17:1683-1695

Lovejoy S, Schertzer D (1990) Multifractals universality classes and satellite and radar measurements of clouds and rain fields. J Geophys Res 95(D3):2021-2034

Lovejoy S, Schertzer D (1995) Multifractals and rain. In: AW Kundzewicz (ed) New Uncertainty Concepts in Hydrology and Hydrological modelling. Cambridge Press, Cambridge, pp 62-103

Olsson J (1995) Limits and characteristics of the multifractal behavior of a high-resolution rainfall time series. Nonlinear Processes Geophys 2(1):23-29

Olsson J, Niemczynowicz J (1996) Multifractal analysis of daily rainfall distributions. J Hydrol 187:29-43

Olsson J, Niemczynowicz J, Berndtsson R (1993) Fractal analysis of high-resolution rainfall time series. $\mathrm{J}$ Geophys Res 98: 23265-23274

Pathirana A, Herath S, Yamada T (2003) Estimating rainfall distributions at high temporal resolutions using a multifractal model. Hydrol Earth Syst Sci 7(5):668-679

Schertzer D, Lovejoy S (1987) Physical modelling and analysis of rain and clouds by scaling multiplicative processes. J Geophys Res 92(D8):9693-9714

Tessier Y, Lovejoy S, Schertzer D (1993) Universal multifractals: theory and observations for rain and clouds. J Appl Meteorol 32:223-250

Tessier Y, Lovejoy S, Hubert P, Schertzer D, Pecknold S (1996) Multifractal analysis and modelling of rainfall and river flows and scaling causal transfer functions. Journal of Geophysical Research 101(D21):26427-26440

Valencia JL, Saa Requejo A, Gascó JM, Tarquis AM (2010) A universal multifractal description applied to precipitation patterns of the Ebro River Basin, Spain. Climate Research 44:17-25 